\title{
Carbon Nanotube/Nanofibers and Graphite Hybrids for Li-Ion Battery Application
}

\author{
Yosuke Nomura, ${ }^{1}$ Ilya V. Anoshkin, ${ }^{2}$ Chikaaki Okuda, ${ }^{3}$ Motoyuki Iijima, ${ }^{4}$ Yoshio Ukyo, ${ }^{3}$ \\ Hidehiro Kamiya, ${ }^{1,5}$ Albert G. Nasibulin, ${ }^{2}$ and Esko I. Kauppinen ${ }^{2}$ \\ ${ }^{1}$ Institute of Engineering, Tokyo University of Agriculture and Technology, 2-24-16 Naka-cho, Koganei, Tokyo 184-8588, Japan \\ ${ }^{2}$ Department of Applied Physics, Aalto University School of Science, P.O. Box 15100, Puumiehenkuja 2, 00076 Espoo, Finland \\ ${ }^{3}$ Toyota Central Research and Development Laboratories, Inc., 41-1 Nagakute Yokomichi, Aichi 480-1192, Japan \\ ${ }^{4}$ Graduate School of Environment and Information Sciences, Yokohama National University, 79-7 Tokiwadai, Hodogaya-ku, \\ Yokohama, Kanagawa 240-8501, Japan \\ ${ }^{5}$ Graduate School of Bio-Applications and Systems Engineering, Tokyo University of Agriculture and Technology, 2-24-16 Naka-cho, \\ Koganei, Tokyo 184-8588, Japan
}

Correspondence should be addressed to Ilya V. Anoshkin; ilya.anoshkin@aalto.fi and Albert G. Nasibulin; albert.nasibulin@aalto.fi

Received 4 November 2013; Revised 9 February 2014; Accepted 9 February 2014; Published 12 March 2014

Academic Editor: Xuedong Bai

Copyright (C) 2014 Yosuke Nomura et al. This is an open access article distributed under the Creative Commons Attribution License, which permits unrestricted use, distribution, and reproduction in any medium, provided the original work is properly cited.

\begin{abstract}
To improve the electrical conductivity of negative electrodes of lithium ion batteries, we applied a direct CVD synthesis of carbon nanomaterials on the surface of graphite particles. To prepare a catalyst, two alternative approaches were utilized: colloidal nanoparticles (NPs) and metal (Ni and Co) nitrate salt precursors deposited on the graphite surface. Both colloidal and precursor systems allowed us to produce carbon nanofibers (CNFs) on the graphite surface with high coverage under the optimum CVD conditions. Electrical measurements revealed that the resistivity of the actual electrodes fabricated from CNFs coated graphite particles was about $40 \%$ lower compared to the original pristine graphite electrodes.
\end{abstract}

\section{Introduction}

Portable power sources have become an essential part of the modern world. Among all possible energy storage batteries, special place belongs to Li-ion batteries (LiBs). The construction of LiBs includes three primary functional parts: the negative and positive electrodes and the electrolyte. The negative electrode (anode) of a conventional $\mathrm{LiB}$ is made from graphite particles (GPs). One of the problems of LiB anodes is high electrical contact resistance between the GPs. To overcome this problem, the dimension of the single elements of the material can be reduced down to the nanosize [1]; alternatively one can utilize some other materials such as amorphous $\mathrm{Si}$, Ge or their various combinations [2]. To improve contact resistance between GPs, additional materials could be used. Carbon nanotubes (CNTs) are one of the foremost contenders for that purpose because of their peculiar structure and unique properties such us high electrical conductivity, tensile strength, flexibility, and chemical stability. Multiwalled [3, 4] or single-walled [5] CNTs have been already demonstrated to be individual anode materials. However, the price and availability of the CNTs at the moment do not allow their wide industrial applications. Carbon nanofibers (CNFs) are much more accessible material with significantly lower price. The main difference between the CNTs and CNFs is their structural arrangement of the graphene layers. CNFs are constructed from stacked cones, cups, or plates of carbon layers, while the layers in the CNTs are always parallel to the tube walls.

In conventional $\mathrm{LiBs}$ to improve the electrical conductivity, the cavities between GPs are filled by carbon black [6]. Nevertheless, CNTs/CNFs are expected to be even more efficient conductive fillers $[7,8]$. However, one of the largest obstacles to create carbon based composites is the difficulty of obtaining a good dispersion of the carbon nanomaterials in a matrix, because they tend to agglomerate and form bundles 


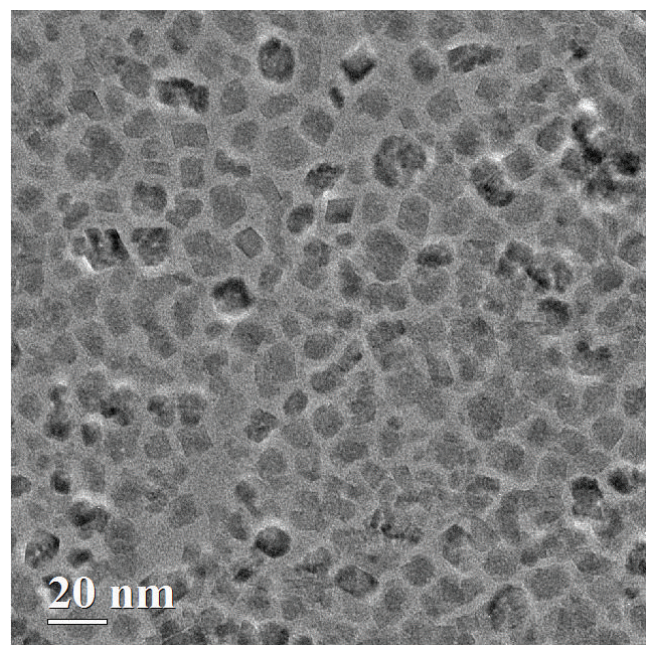

(a)

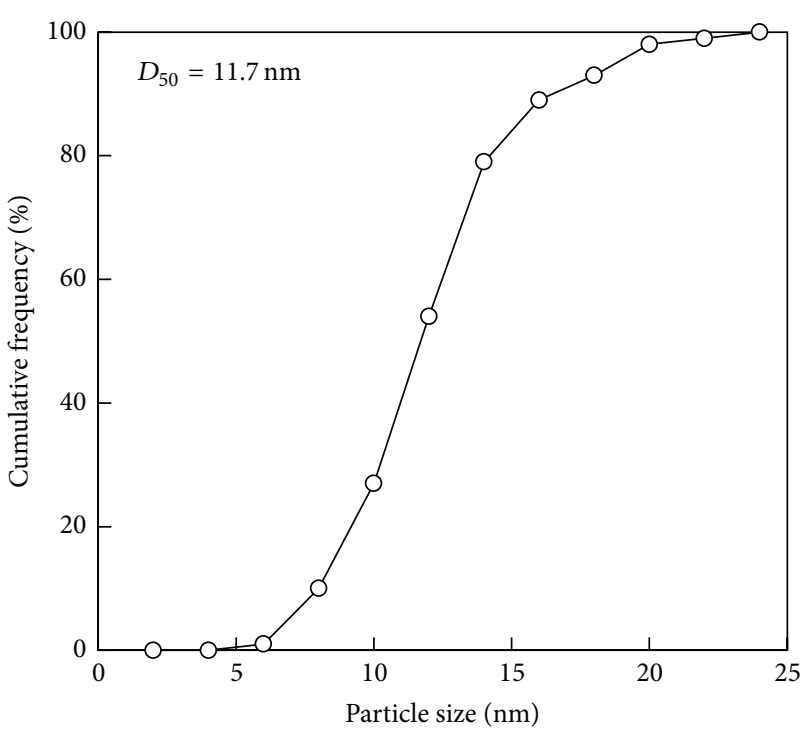

(b)

Figure 1: (a) Typical TEM image of $\mathrm{CoO}$ nanoparticles and (b) cumulative particle size distribution of CoO nanoparticles synthesized by cation exchange reaction.

[9-12]. Even though the powder ball and rotor millings are simple and straightforward, they do not necessarily lead to good mixing and usually shorten and damage the carbon nanomaterials. Moreover, mixing is a highly time- and energy consuming process. Recently, we have proposed an approach of getting a good dispersion of the carbon nanomaterials in a matrix by their direct synthesis on the surface of matrix particles $[13,14]$. Here, we applied this approach to synthesise CNTs/CNFs on the surface of GPs to subsequently improve the electrical performance of the LiB electrode. For that purpose, we utilized two different methods to prepare the catalyst particles on the surface of GPs by cation exchange reaction method and by reduction of metal nitrate salts. Afterwards, we investigated the effect of the synthesis conditions on the morphology and crystallinity of the product. And finally, we carried out electrical measurements of the optimized condition sample and compared with the resistivity of the original graphite electrode.

\section{Experimental}

2.1. Materials and Methods. Graphite particles purchased from Osaka Gas Chemical Co., Ltd. were ca. $20 \mu \mathrm{m}$ in diameter. Oleylamine (OLA, 70\%) and polyethyleneimine $(\mathrm{PEI}, \mathrm{Mw}=1800)$ were received from Fluka and Alfa Aesar, respectively. $\mathrm{Co}\left(\mathrm{NO}_{3}\right)_{2} \cdot 6 \mathrm{H}_{2} \mathrm{O}$ and $\mathrm{Ni}\left(\mathrm{NO}_{3}\right)_{2} \cdot 6 \mathrm{H}_{2} \mathrm{O}$ were supplied by Alfa Aesar. Different solvents used (toluene, ethanol, iso-propanol, etc.) were of analytical grade without any additional purification. Products were observed and analyzed using high resolution scanning electron microscope (SEM: JEOL 7500FA or Zeiss Sigma VP) at low voltages $(1-2 \mathrm{kV})$, transmission electron microscope (TEM: JEOL
2200FS) with two spherical aberration correctors at the acceleration voltages of $80-200 \mathrm{kV}$ [15].

2.2. Colloidal Catalyst Preparation. Cobalt oxide (CoO) nanoparticles (NPs) used as a catalyst for the synthesis of carbon nanomaterials were prepared by cation exchange reaction with $\mathrm{CuO}$ in 1 -octadecene solution at $160^{\circ} \mathrm{C}$ [16]. As-prepared $\mathrm{CoO}$ NPs can be well dispersed in toluene, since they are stabilized by hydrophobic part of the chain of oleylamine (OLA). Typical TEM image of CoO NPs synthesized was shown in Figure 1(a). Median diameter $\left(D_{50}\right)$ of $200 \mathrm{CoO}$ NPs measured from TEM images was determined to be $11.7 \mathrm{~nm}$ as shown in Figure 1(b).

In order to investigate the effect of the type of $\mathrm{CoO}$ suspension on their adsorptions onto the surface of GPs, two different suspensions of CoO NPs were utilized. One of the aforementioned was the $\mathrm{CoO}$ NPs with OLA dispersed in toluene. The other one is $\mathrm{CoO}$ with PEI dispersed in water. To prepare PEI adsorbed CoO NPs, the OLA adsorbed on the surface of $\mathrm{CoO}$ NPs was exchanged to PEI according to the following procedure $[17,18]$. First, 50\% PEI ethanol solution was added to the toluene suspension of as-prepared $\mathrm{CoO}$ and then this mixture was stirred for $30 \mathrm{~min}$. Next, $\mathrm{CoO}$ NPs flocculated by the surfactant exchange were collected by centrifugation $(3000 \mathrm{rpm})$ and then washed by acetone twice to remove soluble OLA. The CoO NPs covered by PEI (unable to be dispersed in acetone) were settled out in the test tube.

In order to adsorb CoO NPs on the graphite particle surface, the toluene suspensions of CoO NPs with OLA were added to GP toluene suspension and stirred for $1 \mathrm{~h}$. Next, the $\mathrm{CoO}$ coated GPs were collected by centrifugation (3000 rpm) and then dried at $105^{\circ} \mathrm{C}$. In the case of $\mathrm{CoO}$ stabilized with PEI in water, $\mathrm{CoO}$ NPs were added to water suspension of 


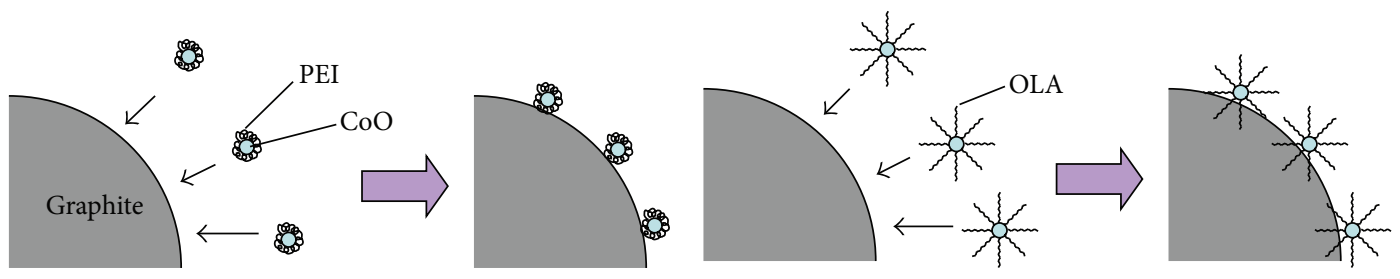

(a)

(b)

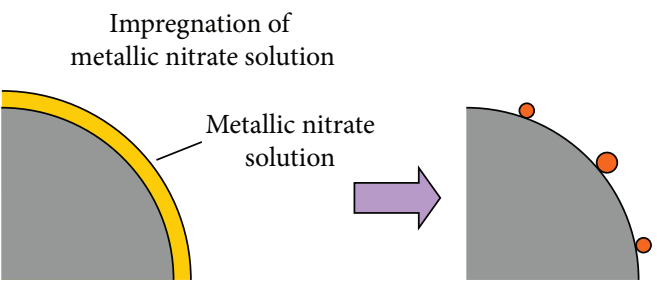

(c)

FIGURE 2: Schematics of catalyst coating methods on graphite surface: (a) $\mathrm{CoO}$ with PEI on pristine GPs (G1) and on GPs treated with $\mathrm{HNO}_{3}$ (G2); (b) CoO with OLA on pristine GPs (G3) and on oxidized GPs (G4); (c) Ni(NO 3$)_{2}$ on pristine GPs (G5) and Co(NO N $_{2}$ on oxidized GPs (G6).

oxidized GPs and then the CoO coated GPs were obtained by the same way as in the case of $\mathrm{CoO}$ with OLA. The weight ratio between $\mathrm{CoO}\left(W_{\mathrm{CoO}}\right)$ and graphite particles $\left(W_{\mathrm{GPs}}\right)$ to form nanoparticle monolayer on the GP surface can be calculated on the basis of the following equation:

$$
\frac{W_{\mathrm{CoO}}}{W_{\mathrm{GPs}}}=\frac{6 \pi}{\sqrt{3}} \frac{D_{\mathrm{CoO}}}{D_{\mathrm{GPs}}} \frac{\rho_{\mathrm{CoO}}}{\rho_{\mathrm{GPs}}},
$$

where $D_{\mathrm{CoO}}$ and $D_{\mathrm{GPs}}$ are diameters of $\mathrm{CoO}$ nanoparticles and GPs; $\rho_{\mathrm{CoO}}$ and $\rho_{\mathrm{GPs}}$ are the bulk densities of $\mathrm{CoO}$ and GPs, respectively. The weight ratio between $\mathrm{CoO}$ NPs and GPs was calculated to be $17.7 \mathrm{mg}$ of $\mathrm{CoO}$ nanoparticles per $1.0 \mathrm{~g}$ of graphite.

In the case of $\mathrm{CoO}$ with $\mathrm{PEI}$, to examine the effect of graphite surface, GPs were treated with $\mathrm{HNO}_{3}$. First, pristine GPs were mixed with $90 \% \mathrm{HNO}_{3}$ solution for $30 \mathrm{~min}$ and then collected by filtration. Next, filtered particles were washed by water and heated up to $550^{\circ} \mathrm{C}$ in a muffle furnace for $15 \mathrm{~min}$ to remove residual $\mathrm{HNO}_{3}$. By this treatment, the surface of GPs was oxidized and covered with functional groups $(-\mathrm{OH},-\mathrm{COOH})$, which could be subsequently used for anchoring $\mathrm{CoO}$ NPs. Hereafter, $\mathrm{CoO}$ with PEI on pristine GPs, $\mathrm{CoO}$ with PEI on $\mathrm{HNO}_{3}$ treated GPs, and $\mathrm{CoO}$ with OLA on pristine GPs are denoted by G1, G2, and G3, respectively. For sample G4, to remove residual OLA and open the surface of $\mathrm{CoO}$, the additional oxidation step for $15 \mathrm{~min}$ at $600^{\circ} \mathrm{C}$ was applied under the air atmosphere.

Schematics of catalyst coating methods on the graphite surface for all samples are summarized in Figure 2. In the cases of G1 and G2, it is likely that catalyst NPs were adsorbed on the graphite surface by electrostatic adsorption in water due to positively charged NPs and negatively charged GPs in water. In the cases of G3 and G4, the electrostatic attractive force could not be acted strongly between GPs and $\mathrm{CoO}$ with OLA, because the surface charge of colloidal particles in aprotic and low-polarity organic solvent (toluene) is generally very low; however wetting the hydrophobic graphite surface by toluene is much higher than in water.

2.3. Catalyst Impregnated from Metal Nitrates. Catalyst on the GP surface was also prepared from metal nitrate solution. Additionally, two kinds of graphite particles were used for the experiment. One was pristine GPs and the other one was oxidized GPs. The oxidized GPs were prepared by introducing graphite particles into a tube furnace heated at $850^{\circ} \mathrm{C}$ in $\mathrm{N}_{2}$ and $\mathrm{O}_{2}$ atmosphere $\left(\sim 5\right.$ vol. $\left.\% \mathrm{O}_{2}\right)$. Then, 5.0 wt. $\% \mathrm{Co}\left(\mathrm{NO}_{3}\right)_{2} \cdot 6 \mathrm{H}_{2} \mathrm{O}$ or 5.0 wt. $\% \mathrm{Ni}\left(\mathrm{NO}_{3}\right)_{2} \cdot 6 \mathrm{H}_{2} \mathrm{O}$ ethanol solution was stirred with GPs for $15 \mathrm{~min}$ and then the particles were filtered and dried at $70^{\circ} \mathrm{C}$. Ni salt solution impregnated GPs and Co based solution impregnated oxidized GPs are denoted by $\mathrm{G} 5$ and G6, respectively.

2.4. Growth of Carbon Nanomaterials. The CVD growth of carbon nanomaterials on the GP surface was carried out at $900^{\circ} \mathrm{C}$ for $15 \mathrm{~min}$ for all samples (except $1 \mathrm{~h}$ for sample G3). During the CVD growth, the flow rate of hydrogen $\left(\mathrm{H}_{2}\right)$ and ethylene $\left(\mathrm{C}_{2} \mathrm{H}_{4}\right)$ was, respectively, maintained at 700 and $13 \mathrm{~cm}^{3} / \mathrm{min}$. Prior to the CVD synthesis, the samples were first exposed to an inert Ar atmosphere $\left(500 \mathrm{~cm}^{3} / \mathrm{min}\right)$, which was replaced by an $\mathrm{H}_{2}$ flow for $5 \mathrm{~min}$ to reduce $\mathrm{CoO}$. After that, $\mathrm{C}_{2} \mathrm{H}_{4}$ was introduced into the $\mathrm{CVD}$ reactor. In some cases, $\mathrm{H}_{2}$ and $\mathrm{C}_{2} \mathrm{H}_{4}$ mixture was directly introduced after the Ar purging. The samples G3 and G4, prior to the synthesis, were first oxidized at $600^{\circ} \mathrm{C}$ for $5 \mathrm{~min}$ in an air atmosphere to clean the $\mathrm{CoO}$ particle surface from the OLA layer.

2.5. Electrical Resistivity Measurement of Negative Electrodes. To examine the electrical property of the electrode, the resistivity of the samples fabricated from the pristine GPs and sample G6 with synthesized carbon nanomaterials was measured by using 4-probe technique. To prepare 


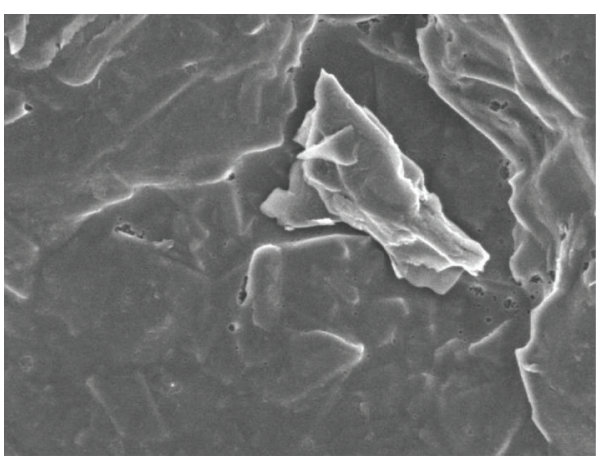

(a)

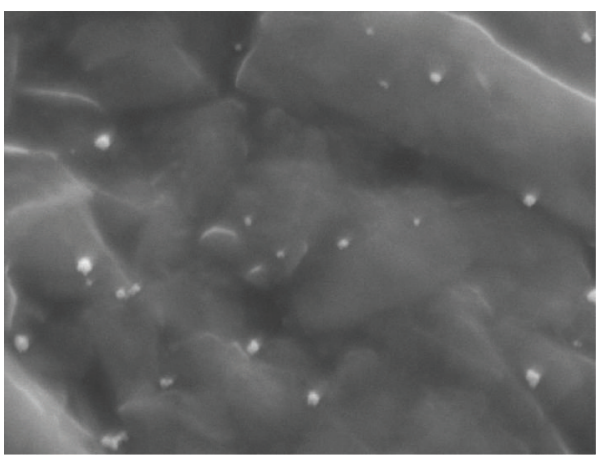

(c)

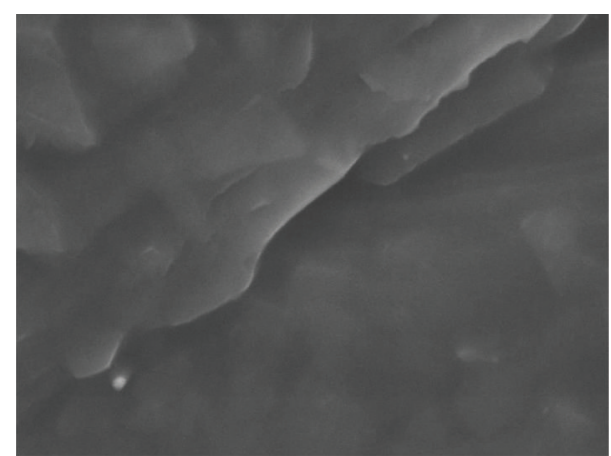

(b)

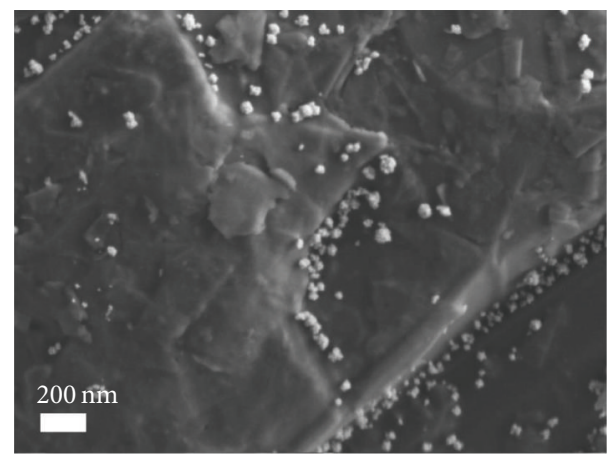

(d)

FIGURE 3: Typical scanning electron microscopy images of (a) pristine GPs; GP surface coated by catalyst in (b) sample G1; (c) sample G2; (d) sample G3.

the electrodes, GPs were mixed with poly(vinylidene fluoride) (binder, PVDF) and $N$-methylpyrrolidone (dispersion medium, NMP) with the weight ratio of the components (GPs: PVDF:NMP) of $9: 1: 10$. Then, the resulting mixture was coated on a $\mathrm{Cu}$ foil and dried at $180^{\circ} \mathrm{C}$.

\section{Results and Discussion}

3.1. Morphology of the Catalyst and Carbon Nanomaterials on GPs. Before the CVD process, the deposition states of colloidal and nitrate catalyst on the graphite surface were observed by SEM. In the case of sample G1, almost no catalyst nanoparticles were observed on the graphite surface (Figure 3(b)). The amount of adsorbed catalyst particles was increased by the treatment with $\mathrm{HNO}_{3}$ (sample G2: Figure $3(\mathrm{c})$ ), since the graphite surface charge in water could be increased by oxidation. On the other hand, in the case of sample G3, many CoO NPs were adsorbed on the graphite surface (Figure 3(d)). This suggested that the degree of an attractive interaction between untreated graphite surface and $\mathrm{CoO}$ with OLA (G3) was higher than for G2. However, in the case of G3, CoO NPs were found as aggregated particles around steps and terraces on graphite surface. In case of metal nitrate system, no visible catalyst particles were observed on the surfaces of both G5 and G6 in SEM.

In order to achieve effective connection between GPs in the actual negative electrodes, both the product morphology and the surface coverage of carbon nanomaterials on the graphite surface are important. After the CVD synthesis, it was found that all the investigated samples were covered by CNFs with the average diameter of about $30 \mathrm{~nm}$ and sometimes by inactive catalyst particles covered by a graphitic layer.

For sample Gl, the surface coverage of the synthesized CNFs was quite lower than that of all other samples. This low coverage is due to the low adsorption of CoO NPs on the graphite surface.

In the case of sample G2, the surface coverage of the generated carbon nanomaterials on graphite surface was significantly higher. This increase in the surface coverage can be explained due to the oxidative treatment of graphite with $\mathrm{HNO}_{3}$. After the $\mathrm{HNO}_{3}$ treatment, the presence of surface functional groups on GPs resulted in more effective CoO NPs adsorption on the surface of GPs. However, even in this case, the surface coverage of synthesized carbon nanomaterials on the graphite surface was quite low.

In the case of samples G3 and G4, the surface coverage of the synthesized carbon nanomaterials on the GPs was higher compared to samples G1 and G2. In comparison to the aqueous colloidal systems (where surfaces of $\mathrm{CoO}$ with PEI and GPs are hydrophilic and hydrophobic, resp.), the toluene based colloidal systems (where both surfaces of $\mathrm{CoO}$ with OLA and GPs are hydrophobic) were found to better suit for the catalyst adsorption. Most likely the attractive interaction between $\mathrm{CoO}$ NPs and the surface of GPs for sample G3 was stronger than that for G2. It should be noted 


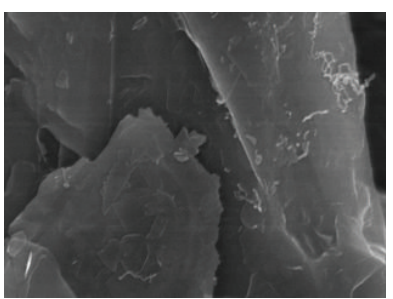

(a)

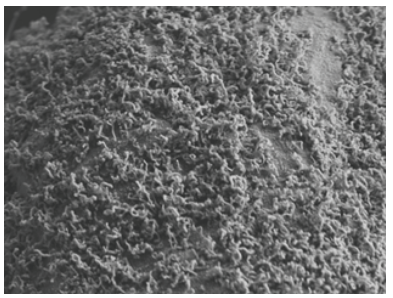

(e)

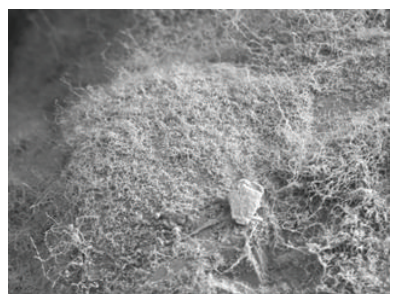

(i)

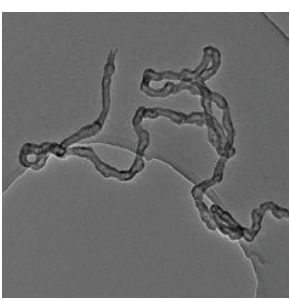

(b)

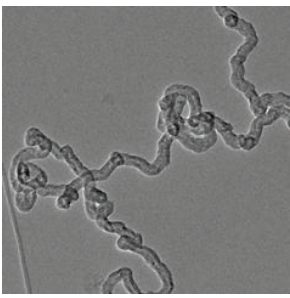

(f)

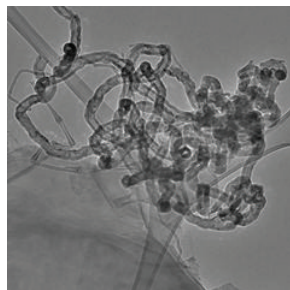

(j)

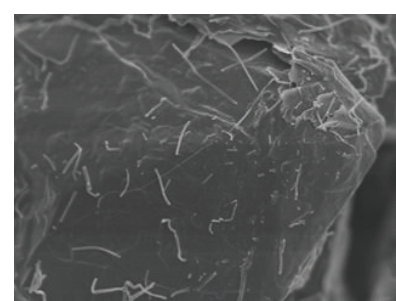

(c)

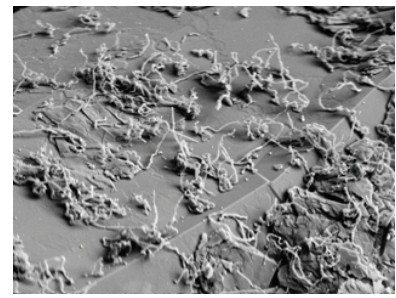

(g)

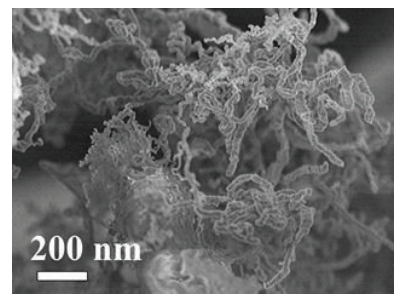

$(\mathrm{k})$

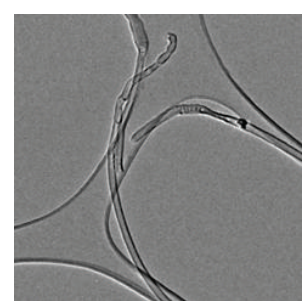

(d)

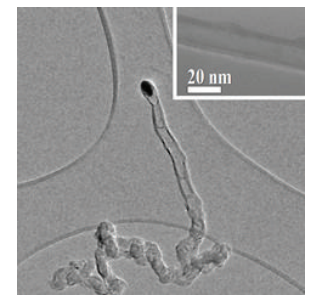

(h)

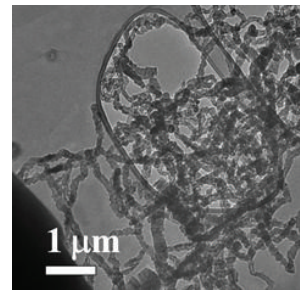

(l)

FIGURE 4: Typical SEM and TEM images of the product synthesized on the surface of GPs in (a, b) sample G1; (c, d) sample G2; (e, f) sample G3; $(g, h)$ sample G4; $(i, j)$ sample G5 and $(k, l)$ sample G6. All SEM and TEM images are, respectively, shown at similar magnifications corresponding to scale bars presented in (l) SEM and (k) TEM images.

that for the $\mathrm{O}_{2}$-oxidized sample G4 in the CVD product, some admixture of multiwalled CNTs was observed (upper left in Figure 4(h)). We believe that it could be explained by catalytic oxidation process of carbon by oxygen during $15 \mathrm{~min}$ annealing at $600^{\circ} \mathrm{C}$. The catalytic activity of transition metal oxides was observed for carbon oxidation [19] and this process could create cavities on the GP surface which prevent sintering metal particles during CVD process and allowed to grow the CNTs with a good crystallinity.

For the nitrate-based catalyst systems (G5 and G6), the surface coverage of the synthesized CNFs on GPs was the highest. It is worth noting that the diameter of generated CNFs was almost equivalent to G3 (colloidal catalyst system) implying the same catalyst particle size.

It is worth noting that the product synthesized of the surface of GPs can be also altered by changing the order of the supplied gases. For instance, when sample G3 was first introduced in an $\mathrm{H}_{2}$ atmosphere, which was subsequently switched to the $\mathrm{H}_{2}$ and $\mathrm{C}_{2} \mathrm{H}_{4}$ mixture, no tubular nanomaterials were produced on the graphite surface. TEM images of the catalyst particles indicated that they were covered with the graphitic layers (Figure 5(a)) and the surface of GPs was etched by the catalyst NPs similarly to $[19,20]$. In the previous studies, the catalyst particle dissociates molecular hydrogen, which reacts with carbon $[8,20]$. The resulting etched channel generated on the graphite surface is shown in Figure 5(b).
However, when the $\mathrm{H}_{2}$ and $\mathrm{C}_{2} \mathrm{H}_{4}$ mixture was introduced right after the inert $\mathrm{Ar}$ atmosphere, the product considerable changed (Figure 4(e)). High coverage of the GPs by the CNFs was obtained. Similar results were found for all of other samples. To get the highest coverage of the CNFs on the GP surface, the mixture of $\mathrm{H}_{2}$ and $\mathrm{C}_{2} \mathrm{H}_{4}$ should be introduced right after the Ar purging process.

3.2. Electrical Conductivity of Prepared Electrodes. Finally, negative electrodes were prepared from the pristine graphite and one of the CNFs coated GP samples. Sample G6 was selected for testing, since this sample contained the highest coverage of CNFs. Figure 6 shows the relationship between the electrical resistivity of the negative electrodes and the load. The electrical resistivity of both electrodes exponentially decreased with increasing the loading force. Moreover, these results revealed that the electrical resistivity of the electrode prepared from modified GP surface was lower than that of pristine graphite in the pressure range from 2 to $100 \mathrm{~kg} / \mathrm{cm}^{2}$. It was found that the reduction in resistivity was about $40 \%$ at $50 \mathrm{~kg} / \mathrm{cm}^{2}$. This notable decrease in the electrical resistivity of electrode prepared from sample G6 can be explained by higher efficiency in the electrical connection between the GPs, which was caused by produced CNFs.

The battery power performance can be discussed based on the relationship between the electrical resistivity of the 


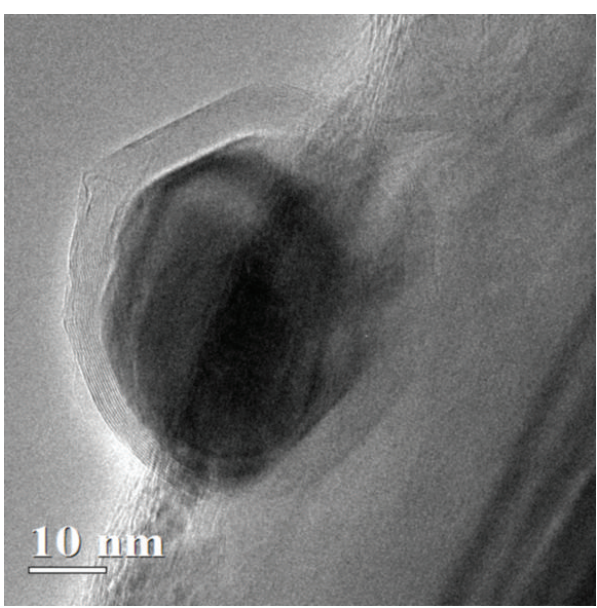

(a)

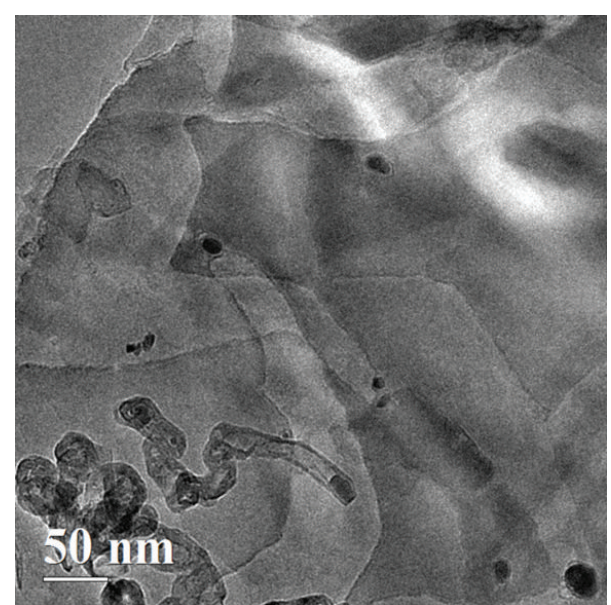

(b)

FIGURE 5: Typical TEM images of (a) a catalyst particle coated by graphitic layer and (b) graphite surface with an etched track by catalyst NPs.

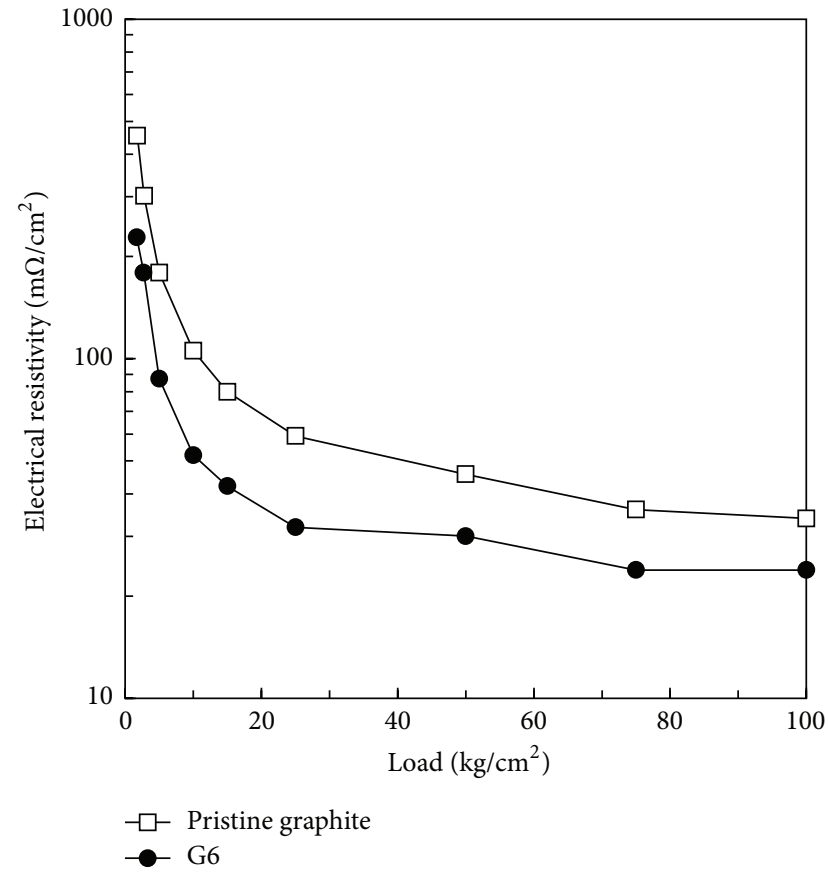

FIGURE 6: Electrical resistivity of Li-ion battery negative electrode prepared from pristine graphite and sample G6 with synthesized CNFs as a function of the load pressure.

electrodes and the load. In general, to reduce the contact resistance between GPs in a negative electrode, a high loading force is usually applied, because to lower the contact resistance, the larger contact area between GPs are required. However, higher electrode density causes lower Li-ion diffusion in the electrode during the battery operation, since the diffusion is limited by narrow pore sizes, that is, by high density. Therefore, the CNFs coated GPs are believed to be advantageous over the pristine GPs at similar loads.

\section{Conclusion}

To improve the electrical conductivity of GPs in the Li-ion negative electrodes, we directly synthesized carbon nanomaterials on the surface of GPs. To prepare the catalyst, two alternative approaches were utilized: colloidal nanoparticles (NPs) and metal (Ni and $\mathrm{Co}$ ) nitrate salt precursors deposited on the graphite surface. The most suitable conditions for the synthesis of CNFs and CNTs with the highest coverage were found at $900^{\circ} \mathrm{C}$ with cobalt nitrate precursor impregnated oxidized GPs, when the mixture of $\mathrm{H}_{2}$ and $\mathrm{C}_{2} \mathrm{H}_{4}$ was introduced right after Ar purging process. The negative electrode prepared from this optimum sample showed noticeable resistivity decrease down to $\sim 40 \%$ at $50 \mathrm{~kg} / \mathrm{m}^{2}$ loaded pressure.

\section{Conflict of Interests}

The authors declare that there is no conflict of interests regarding the publication of this paper.

\section{Acknowledgments}

This study was supported by the "Strategic International Cooperative Program of Japan Science and Technology Agency and Finnish Funding Agency for Technology and Innovation" and the "Strategic Young Researcher Overseas Visits Program for Accelerating Brain Circulation of Japan Society for Promotion of Science."

\section{References}

[1] K. T. Lee and J. Cho, "Roles of nanosize in lithium reactive nanomaterials for lithium ion batteries," Nano Today, vol. 6, no. 1, pp. 28-41, 2011.

[2] R. Mukherjee, R. Krishnan, T. M. Lu, and N. Koratkar, "Nanostructured electrodes for high-power lithium ion batteries," Nano Energy, vol. 1, no. 4, pp. 518-533, 2012. 
[3] E. Frackowiak, S. Gautier, H. Gaucher, S. Bonnamy, and F. Beguin, "Electrochemical storage of lithium multiwalled carbon nanotubes," Carbon, vol. 37, no. 1, pp. 61-69, 1999.

[4] H.-C. Shin, M. Liu, B. Sadanadan, and A. M. Rao, "Electrochemical insertion of lithium into multi-walled carbon nanotubes prepared by catalytic decomposition," Journal of Power Sources, vol. 112, no. 1, pp. 216-221, 2002.

[5] B. Gao, C. Bower, J. D. Lorentzen et al., "Enhanced saturation lithium composition in ball-milled single-walled carbon nanotubes," Chemical Physics Letters, vol. 327, no. 1-2, pp. 69-75, 2000.

[6] W. Guoping, Z. Qingtang, Y. Zuolong, and Q. MeiZheng, "The effect of different kinds of nano-carbon conductive additives in lithium ion batteries on the resistance and electrochemical behavior of the $\mathrm{LiCoO} 2$ composite cathodes," Solid State Ionics, vol. 179, no. 7-8, pp. 263-268, 2008.

[7] X.-M. Liu, Z. D. Huang, S. W. Oh et al., "Carbon nanotube (CNT)-based composites as electrode material for rechargeable Li-ion batteries: a review," Composites Science and Technology, vol. 72, no. 2, pp. 121-144, 2012.

[8] C. de las Casas and W. Li, "A review of application of carbon nanotubes for lithium ion battery anode material," Journal of Power Sources, vol. 208, pp. 74-85, 2012.

[9] A. Yazdanbakhsh and Z. Grasley, "The theoretical maximum achievable dispersion of nanoinclusions in cement paste," Cement and Concrete Research, vol. 42, no. 6, pp. 798-804, 2012.

[10] A. Yazdanbakhsh, Z. Grasley, B. Tyson, and R. K. Abu AlRub, "Dispersion quantification of inclusions in composites," Composites Part A: Applied Science and Manufacturing, vol. 42, no. 1, pp. 75-83, 2011.

[11] L. Jiang, Z. Li, G. Fan, L. Cao, and D. Zhang, “The use of flake powder metallurgy to produce carbon nanotube (CNT)/aluminum composites with a homogenous CNT distribution," Carbon, vol. 50, no. 5, pp. 1993-1998, 2012.

[12] L. Jiang, G. Fan, Z. Li et al., "An approach to the uniform dispersion of a high volume fraction of carbon nanotubes in aluminum powder," Carbon, vol. 49, no. 6, pp. 1965-1971, 2011.

[13] A. G. Nasibulin, S. D. Shandakov, L. I. Nasibulina et al., "A novel cement-based hybrid material," New Journal of Physics, vol. 11, Article ID 023013, 2009.

[14] L. I. Nasibulina, T. S. Koltsova, T. Joentakanen et al., "Direct synthesis of carbon nanofibers on the surface of copper powder," Carbon, vol. 48, no. 15, pp. 4559-4562, 2010.

[15] H. Jiang, J. Ruokolainen, N. Young et al., "Performance and early applications of a versatile double aberration-corrected JEOL2200FS FEG TEM/STEM at Aalto University," Micron, vol. 43, no. 4, pp. 545-550, 2012.

[16] J. Ning, G. Xiao, L. Wang, B. Zou, B. Liu, and G. Zou, "Facile synthesis of magnetic metal ( $\mathrm{Mn}, \mathrm{Fe}, \mathrm{Co}$, and $\mathrm{Ni}$ ) oxides nanocrystals via a cation-exchange reaction," Nanoscale, vol. 3, no. 2, pp. 741-745, 2011.

[17] S. Sun, S. Anders, T. Thomson et al., "Controlled synthesis and assembly of FePt nanoparticles," Journal of Physical Chemistry B, vol. 107, no. 23, pp. 5419-5425, 2003.

[18] W. Wu, Q. He, and C. Jiang, "Magnetic iron oxide nanoparticles: synthesis and surface functionalization strategies," Nanoscale Research Letters, vol. 3, no. 11, pp. 397-415, 2008.

[19] F. Schäffel, J. H. Warner, A. Bachmatiuk et al., "Shedding light on the crystallographic etching of multi-layer graphene at the atomic scale," Nano Research, vol. 2, no. 9, pp. 695-705, 2009.
[20] F. Schäffel, J. H. Warner, A. Bachmatiuk et al., "On the catalytic hydrogenation of graphite for graphene nanoribbon fabrication," Physica Status Solidi (B) Basic Research, vol. 246, no. 11-12, pp. 2540-2544, 2009. 

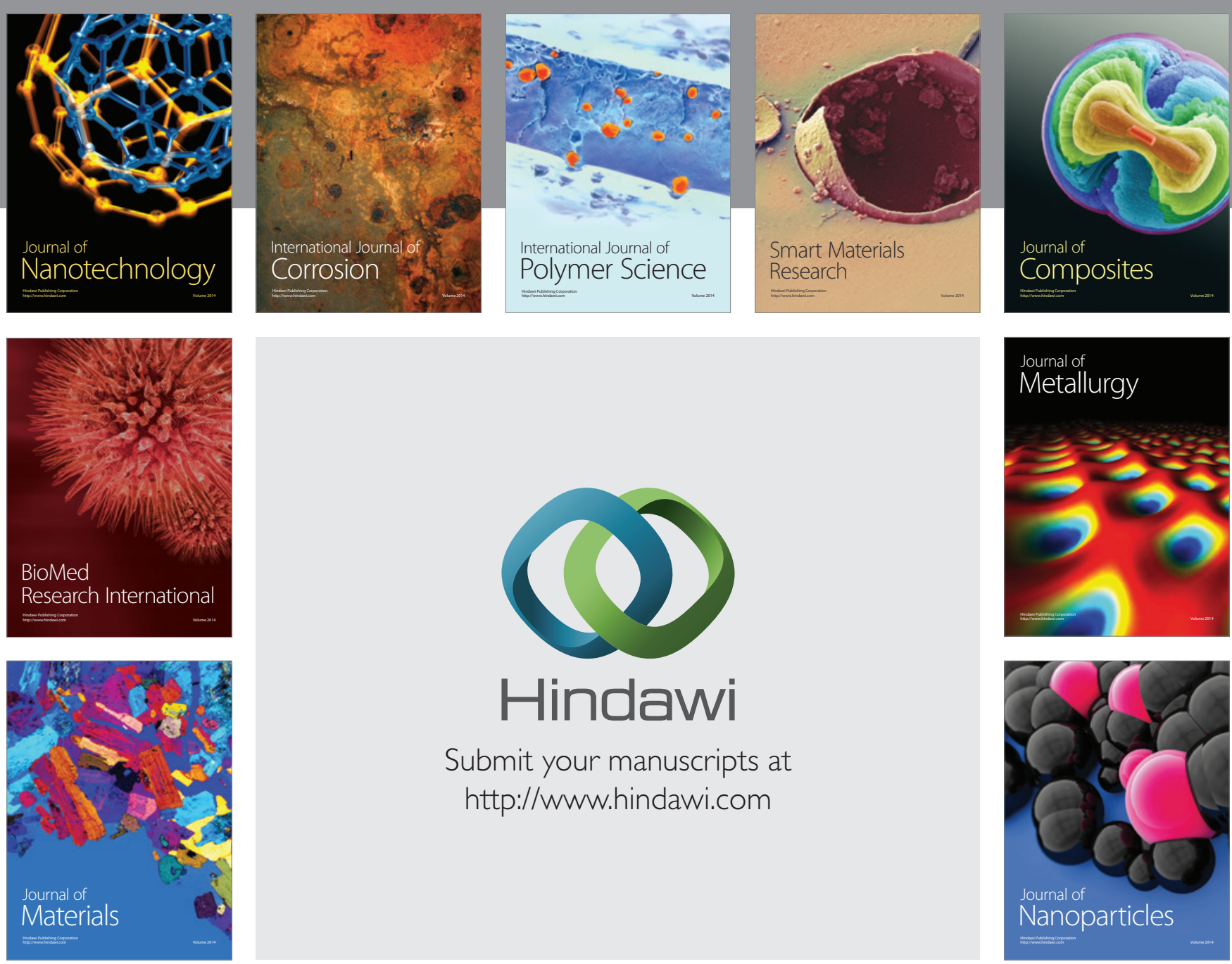

Submit your manuscripts at http://www.hindawi.com
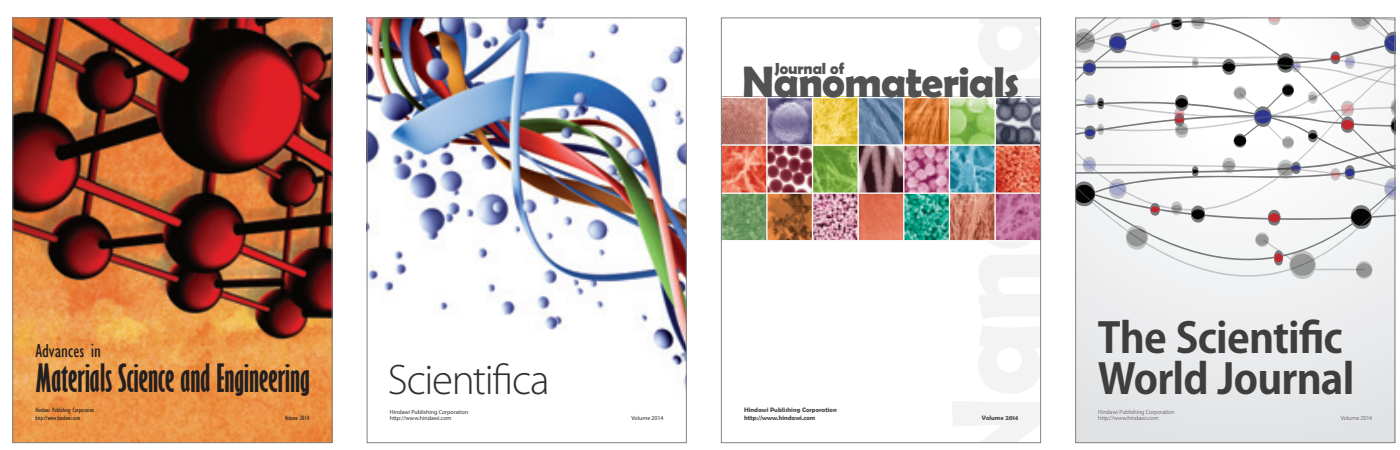

\section{The Scientific World Journal}
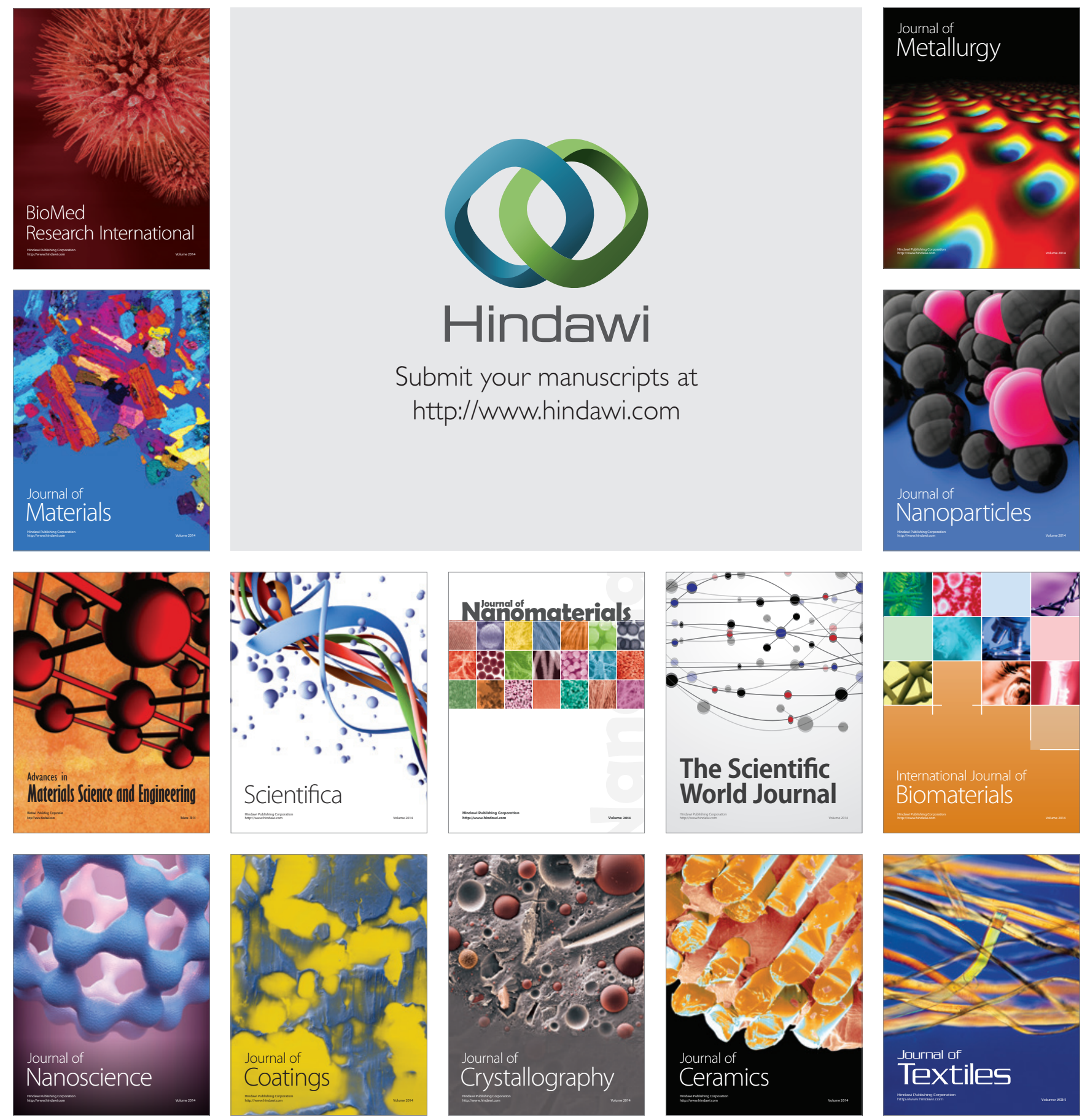